\title{
Pressure-stabilized unconventional stoichiometric yttrium sulfides
}

\author{
Ju Chen, ${ }^{1}$ Wenwen Cui $\odot,{ }^{1, *}$ Kun Gao, ${ }^{1}$ Jian Hao, ${ }^{1}$ Jingming Shi, ${ }^{1}$ and Yinwei Li $\odot{ }^{1,2, \uparrow}$ \\ ${ }^{1}$ Laboratory of Quantum Functional Materials Design and Application, School of Physics and Electronic Engineering, \\ Jiangsu Normal University, Xuzhou 221116, China \\ ${ }^{2}$ Shandong Key Laboratory of Optical Communication Science and Technology, School of Physical Science and Information Technology of \\ Liaocheng University, Liaocheng 252059, China
}

(Received 12 October 2020; revised 1 December 2020; accepted 14 December 2020; published 29 December 2020)

\begin{abstract}
Yttrium sulfides are found to exhibit rich phase diagram and diverse properties under pressures. Here, we systematically investigate the stability of binary Y-S system up to $50 \mathrm{GPa}$ using first-principles swarm-intelligence structure search. We identify seven hitherto unknown stoichiometries $\left(\mathrm{Y}_{7} \mathrm{~S}_{6}, \mathrm{Y}_{6} \mathrm{~S}_{5}, \mathrm{Y}_{7} \mathrm{~S}_{8}, \mathrm{Y}_{6} \mathrm{~S}_{7}, \mathrm{Y}_{5} \mathrm{~S}_{6}, \mathrm{Y}_{3} \mathrm{~S}_{2}\right.$, and $\mathrm{YS}_{3}$ ) that are energetically stable with respect to the decomposition into elemental components. All phases exhibit metallic nature, of which $\mathrm{YS}_{3}$ shows potential superconductivity with estimated $T_{c}$ of $18.5 \mathrm{~K}$ at $50 \mathrm{GPa}$. The $T_{c}$ in $\mathrm{YS}_{3}$ is comparable to the $17 \mathrm{~K}$ superconductivity in elements yttrium (89.3 GPa) and sulfur (160 GPa), but could be achieved at much lower pressure. The high superconductivity is attributed to the high electronic density of states from $\mathrm{S}$ atoms at Fermi level. Additionally, $\mathrm{Y}_{3} \mathrm{~S}_{2}$ is predicted to be a layer-structured magnetic electride with magnetic moment of $0.5 \mu \mathrm{B}$ per formula unit at $6 \mathrm{GPa}$, which transforms to a three-dimensional phase with weak superconductivity around $17 \mathrm{GPa}$.
\end{abstract}

DOI: 10.1103/PhysRevResearch.2.043435

\section{INTRODUCTION}

Many efforts have been devoted to investigating high- $T_{c}$ superconductors at extreme conditions, where high pressure is a powerful tool to stabilize the compounds that are inaccessible at ambient pressure. Recently, several theory-initiated discoveries of high- $T_{c}$ hydrides superconductors, such as $203 \mathrm{~K}$ in $\mathrm{H}_{3} \mathrm{~S}$ at $150 \mathrm{GPa}$ [1-3], $260 \mathrm{~K}$ in $\mathrm{LaH}_{10}$ at $170 \mathrm{GPa}$ [4-7], and $224 \mathrm{~K}$ in $\mathrm{YH}_{6}$ at $120 \mathrm{GPa}$ [8-11], have brought about a new era of superconductors. However, because the superconductivities of these hydrides are only retained at extremely high pressure (>100 GPa), practical applications are a big challenge. Therefore, it is highly desirable to reduce the pressure at which superconductivity emerges.

Elements yttrium (Y) and sulfur (S) can transform to superconductors with same $T_{c}$ of $17 \mathrm{~K}$ at $89.3 \mathrm{GPa}$ and $160 \mathrm{GPa}$ [12-14], respectively. The research on their binary yttrium sulfides is particularly fascinating, with four known phases (YS, $\mathrm{Y}_{3} \mathrm{~S}_{4}, \mathrm{Y}_{2} \mathrm{~S}_{3}$, and $\mathrm{YS}_{2}$ ) available at ambient or high pressure [15-17]. As yttrium and sulfur have +3 and -2 oxidation states, respectively, the conventional stoichiometric $\mathrm{Y}_{2} \mathrm{~S}_{3}$ is a semiconductor at ambient pressure. Studies show that the increased $\mathrm{Y}$ atomic fraction can significantly regulate the electronic properties, and all Y-S compounds with higher

\footnotetext{
*wenwencui@jsnu.edu.cn

†yinwei_li@jsnu.edu.cn

Published by the American Physical Society under the terms of the Creative Commons Attribution 4.0 International license. Further distribution of this work must maintain attribution to the author(s) and the published article's title, journal citation, and DOI.
}

$\mathrm{Y}$ ratio as compared to $\mathrm{Y}_{2} \mathrm{~S}_{3}$ show metallic nature. In particular, experiments show that the NaCl-type YS is a superconductor with $T_{c}$ of $2 \mathrm{~K}$ at ambient pressure, which vanishes when transforming to the CsCl-type structure at $50 \mathrm{GPa}$ [18-20]. Additionally, weak superconductivity $(3.6 \mathrm{~K})$ is also observed in the $\mathrm{Th}_{3} \mathrm{P}_{4}$-type $\mathrm{Y}_{3} \mathrm{~S}_{4}$ that was synthesized at high pressure and high temperature [21]. However, $\mathrm{YS}_{2}$ with higher $\mathrm{S}$ atomic fraction retains the semiconducting character.

Pressure is an effective tool to stabilize compounds with unconventional stoichiometries that possess exotic properties [22-26]. Here, we extensively investigate the crystal structures of various stoichiometric $\mathrm{Y}_{x} \mathrm{~S}_{y}(x, y=1-8)$ at 0 and $50 \mathrm{GPa}$. In addition to the known compounds, the predictions uncover seven new thermodynamically stable compounds with stoichiometries $\mathrm{Y}_{7} \mathrm{~S}_{6}, \mathrm{Y}_{6} \mathrm{~S}_{5}, \mathrm{Y}_{7} \mathrm{~S}_{8}, \mathrm{Y}_{6} \mathrm{~S}_{7}, \mathrm{Y}_{5} \mathrm{~S}_{6}, \mathrm{Y}_{3} \mathrm{~S}_{2}$, and $\mathrm{YS}_{3}$. Interestingly, an $\mathrm{S}$-rich $\mathrm{YS}_{3}$ is predicted to be a superconductor with $T_{c}$ of $18 \mathrm{~K}$ at $50 \mathrm{GPa}$, close to that of elements $\mathrm{Y}$ and $\mathrm{S}$, but could be achieved at much lower pressure. Additionally, the layer-structured Y-rich $\mathrm{Y}_{3} \mathrm{~S}_{2}$ is found to be an electride with magnetism. The layer structure of $\mathrm{Y}_{3} \mathrm{~S}_{2}$ transforms to a three-dimensional structure at $17 \mathrm{GPa}$, which shows weak superconductivity of $3 \mathrm{~K}$ at $20 \mathrm{GPa}$.

\section{COMPUTATIONAL DETAILS}

The crystal structure searches on $\mathrm{Y}_{x} \mathrm{~S}_{y}(x, y=1-8)$ were performed by using crystal structure analysis by particle swarm optimization (CALYPSO) methodology [27-29], whose validity has been verified by correctly predicting predicting various crystal structures under high pressure [30-34]. In structure prediction runs, each generation contains 50 structures. For the first generation, the crystal structures are generated randomly with some symmetry constraints and 
subsequent optimizations. Then in the following generations, $60 \%$ of the structures with the lowest enthalpy through the particle swarm optimization algorithms are selected from the previous generation plus the $40 \%$ that are generated randomly to form the next generation. The structure search can be stopped when $\approx 1000$ structures were generated and there are no new structures with lower energy. Structure relaxations and electronic structure calculations were performed using density functional theory within the Perdew-Burke-Ernzerhof (PBE) functional [35] in the framework of the all-electron projector augmented wave (PAW) method [36] as implemented in the VASP code [37]. The Y $4 s^{2} 4 p^{6} 5 s^{2} 4 d^{1}$ and $\mathrm{S} 3 s^{2} 3 p^{4}$ electrons were treated explicitly in all of the calculations. The plane-wave basis set with energy cutoffs of 700 and $1000 \mathrm{eV}$ were employed for structure prediction and precise optimization, respectively. The $k$-point grids with density of $0.20 \AA^{-1}$ were generated using the the Monkhorst-Pack scheme [38]. The $k$ meshes and energy cutoffs have been chosen to ensure that all the enthalpy calculations are well converged to 1 $\mathrm{meV} /$ atom. The electron-phonon coupling calculations were performed within the framework of the linear response theory through QUANTUM ESPRESSO code [39]. Ultrasoft pseudopotentials for $\mathrm{Y}$ and $\mathrm{S}$ were used with a kinetic cutoff energy of $100 \mathrm{Ry}$. The critical superconducting temperature, $T_{c}$, has been estimated using the Allen-Dynes modified McMillan equation [40]. The crystal structures and electron localization function (ELF) were plotted using VESTA software [41].

\section{RESULTS AND DISCUSSION}

An extensive variable-composition crystal structure search for $\mathrm{Y}_{x} \mathrm{~S}_{y}(x, y=1-8)$ was performed at 0 and $50 \mathrm{GPa}$. The maximum number of atoms in the simulation cell for each composition is no more than 24 atoms. The formation enthalpies of all considered Y-S compositions were calculated to construct the convex hulls, where a point lying on the tie line corresponds to a thermodynamically stable phase [Figs. 1(a) and 1(b)]. For those stoichiometries close to the convex hull, a fixed-composition structure search with a maximum four formula units (f.u.) per simulation cell was carried out to obtain the most stable structures. For those stable Y-S compounds deduced from the convex hulls, the relative enthalpies with respect to two neighboring stable compounds or elements were then calculated as functions of pressure in range of 0-60 GPa to obtain the phase diagrams as shown in Fig. 1(c). As expected, the known four compounds lie on the convex hull at ambient pressure, three ( $\mathrm{YS}, \mathrm{Y}_{3} \mathrm{~S}_{4}$, and $\mathrm{YS}_{2}$ ) of which stay thermodynamically stable up to $50 \mathrm{GPa}$ [Fig. 1(b)]. However, the conventional stoichiometry $\mathrm{Y}_{2} \mathrm{~S}_{3}$ possessing the lowest formation enthalpy at ambient pressure decomposes into $\mathrm{Y}_{3} \mathrm{~S}_{4}$ and $\mathrm{YS}_{2}$ at $8 \mathrm{GPa}$ [Fig. 1(c)]. In addition to the known stoichiometries, the structure searches at ambient pressure identify five new thermodynamically stable compounds with stoichiometries $\mathrm{Y}_{6} \mathrm{~S}_{5}, \mathrm{Y}_{7} \mathrm{~S}_{6}, \mathrm{Y}_{7} \mathrm{~S}_{8}, \mathrm{Y}_{6} \mathrm{~S}_{7}$, and $\mathrm{Y}_{5} \mathrm{~S}_{6}$. Among them, $\mathrm{Y}_{6} \mathrm{~S}_{5}$ and $\mathrm{Y}_{7} \mathrm{~S}_{6}$ are constructed by edge-sharing $\mathrm{SY}_{6}$ octahedrons, while the other three by edge-sharing $\mathrm{YS}_{6}$ octahedrons (please see the Supplemental Material, Fig. S1 [46]). Additionally, all five compounds are metallic with electronic density of states at Fermi level dominated by $\mathrm{Y}$ atoms
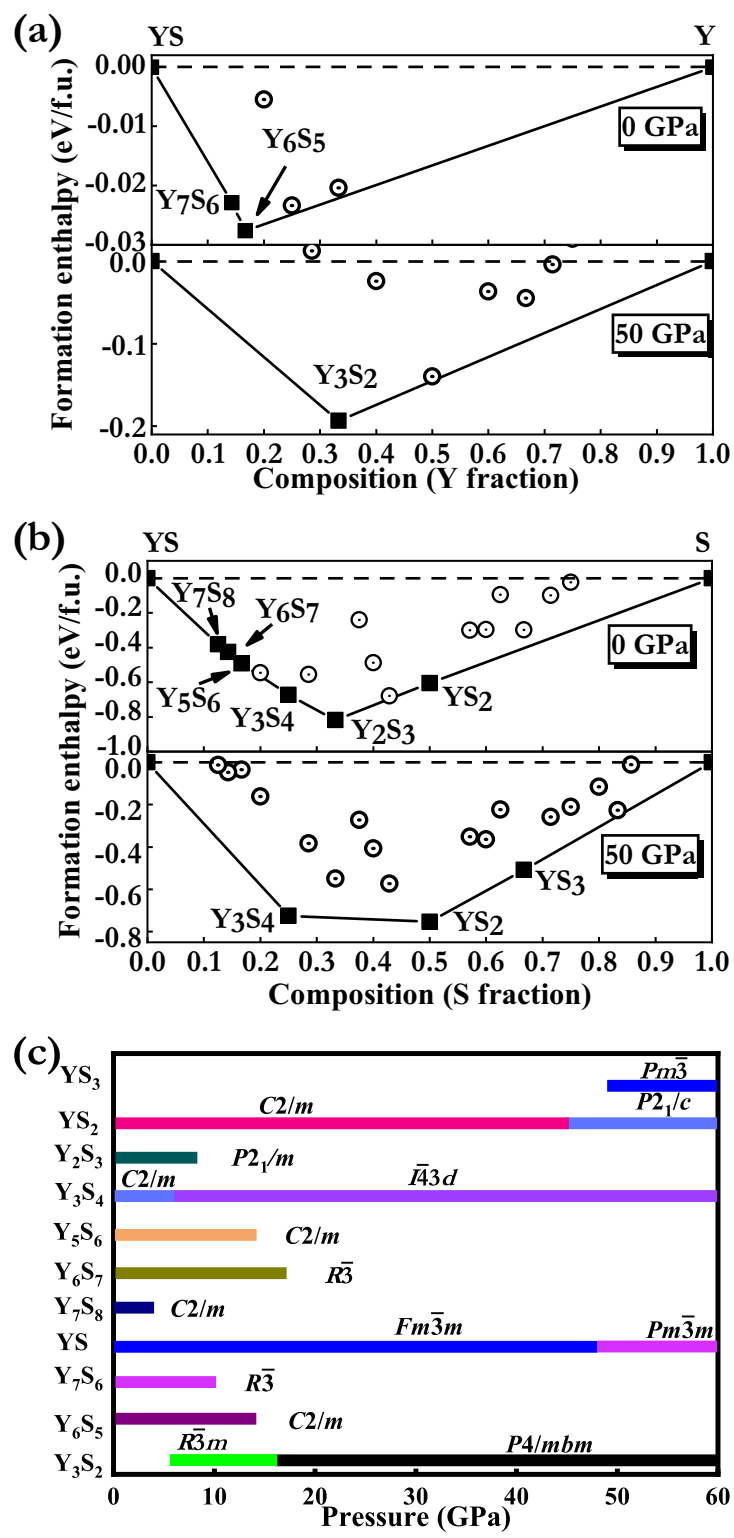

FIG. 1. The calculated formation enthalpies of various Y-S compounds with respect to $\mathrm{YS}+\mathrm{Y}$ (a) and $\mathrm{YS}+\mathrm{S}(\mathrm{b})$ at 0 and $50 \mathrm{GPa}$. The referenced structures of $\mathrm{Y}$ and $\mathrm{S}$ are taken from Refs. [18,21, 42-45]. Panel (c) shows the phase diagrams of all phases as functions of pressure.

(Fig. S2). Phonon calculations confirm that all of them are dynamically stable without any negative frequencies (Fig. S3).

Structure searches at $50 \mathrm{GPa}$ predict two new compounds, a Y-rich $\mathrm{Y}_{3} \mathrm{~S}_{2}$ and an S-rich $\mathrm{YS}_{3}$. Calculations show that $\mathrm{Y}_{3} \mathrm{~S}_{2}$ become energetically stable at pressure as low as $6 \mathrm{GPa}$. The low-pressure phase of $\mathrm{Y}_{3} \mathrm{~S}_{2}$ adopts hexagonal $R \overline{3} \mathrm{~m}$ structure, which is a layered structure consisting of edge-sharing $\mathrm{SY}_{6}$ octahedrons [Fig. 2(a)]. Interestingly, the $\mathrm{Y}$ framework in $\mathrm{Y}_{3} \mathrm{~S}_{2}$ is isostructural to high-pressure Sm-type $\mathrm{Y}$ phase $[43,44]$. Under compression, the layered $R \overline{3} m$ is overtaken by a three-dimensional tetragonal $P 4 / \mathrm{mbm}$ structure at $17 \mathrm{GPa}$ [Figs. 1(c) and 2(c)]. The coordination number of $\mathrm{S}$ increases from 6 in the $R \overline{3} m$ structure to 8 in the $P 4 / m b m$ structure, resulting in the formation of face-sharing $\mathrm{SY}_{8}$ polyhedrons 
(a)
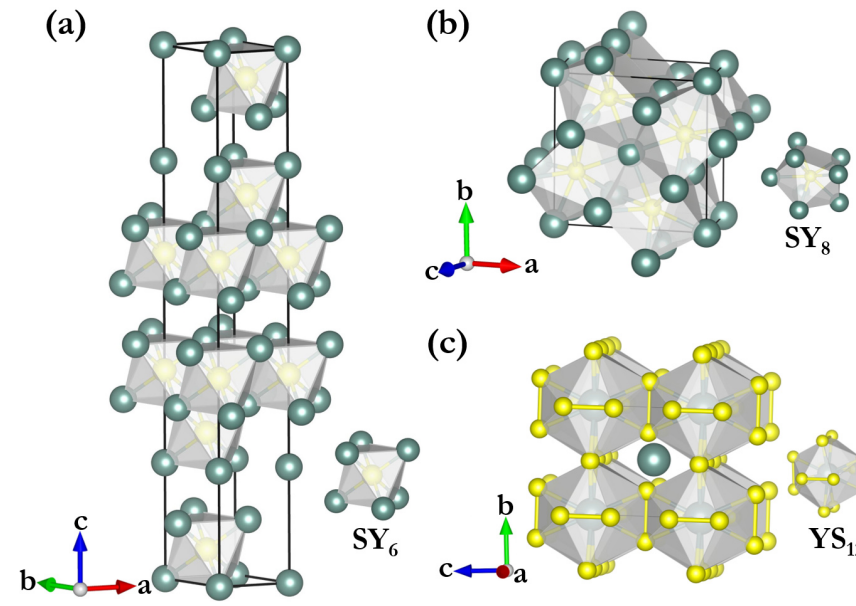

(c)
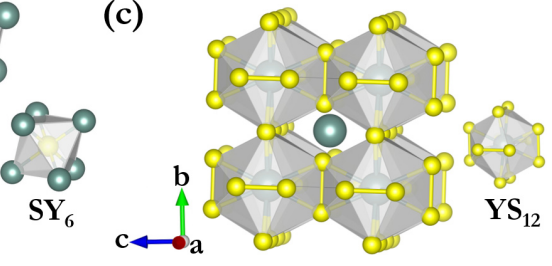

FIG. 2. The schematics of the $R \overline{3} \mathrm{~m}$ structure (a) and the $P 4 / \mathrm{mbm}$ structure (b) of $\mathrm{Y}_{3} \mathrm{~S}_{2}$, and the $P m \overline{3}$ structure of $\mathrm{YS}_{3}$ (c). Polyhedrons beside the structures represent the building blocks. The big gray-green and small yellow spheres represent $\mathrm{Y}$ and $\mathrm{S}$ atoms, respectively.

[Fig. 2(b)]. $\mathrm{YS}_{3}$ possessing the cubic $P m \overline{3}$ structure becomes energetically stable at $48 \mathrm{GPa}$ [Fig. 1(c)]. Unlike other Y-S compounds, $\mathrm{S}_{2}$ dimers are formed in $\mathrm{YS}_{3}$ with $\mathrm{S}$-S bond distance of $2.19 \AA$ (50 GPa), close to that $\left(\approx 2.17 \AA\right.$ ) of $\mathrm{S}_{8}$ ring-shaped molecules in phase I at $0 \mathrm{GPa}$ [47]. Electron localization function (ELF) calculation reveals the covalent bond nature of $S_{2}$ dimer in view of the electron localization between two $\mathrm{S}$ atoms. The $P m \overline{3}$ structure contains two inequivalent $\mathrm{Y}$ atoms that locate at the sites of a bcc lattice. Each $\mathrm{Y}$ atom occupying the bcc vertexes is surrounded by six $\mathrm{S}_{2}$ dimers, forming a $\mathrm{YS}_{12}$ icosahedron with Y-S bond distance of $2.62 \AA$ at $50 \mathrm{GPa}$. Another $\mathrm{Y}$ atom locates in the center of eight $\mathrm{YS}_{12}$ icosahedrons with a Y-S bond distance of $2.70 \AA$ A. Hereafter, the discussions will be focused on the two pressure-stabilized compounds, $\mathrm{Y}_{3} \mathrm{~S}_{2}$ and $\mathrm{YS}_{3}$, both of which are shown to possess exotic properties.

Both $\mathrm{Y}_{3} \mathrm{~S}_{2}$ and $\mathrm{YS}_{3}$ exhibit metallic features with bands crossing the Fermi level [Figs. 3(a)-3(c)]. For $\mathrm{Y}_{3} \mathrm{~S}_{2}$, the DOS around the Fermi level in both the $R \overline{3} \mathrm{~m}$ and $P 4 / \mathrm{mbm}$ structures are dominated by electrons from $\mathrm{Y}$ atoms, while the contribution from the $\mathrm{S}$ atoms is negligible. Interestingly, the layered $R \overline{3} m-Y_{3} \mathrm{~S}_{2}$ is a ferromagnetic metal in view of the different band structures and projected DOS of spin-up and spin-down electrons. The total magnetic moment of $\mathrm{Y}_{3} \mathrm{~S}_{2}$ is calculated to be $\approx 0.5 \mu B$ per formula unit at $6 \mathrm{GPa}$ and stays unchanged up to $17 \mathrm{GPa}$, the maximum stable pressure, which is mainly from the $\mathrm{Y} 4 d$ orbitals. Interestingly, ELF results show that $\mathrm{Y}_{3} \mathrm{~S}_{2}$ is an electride with dumbbell-like electrons localizing in the interstices of two neighboring $\mathrm{SY}_{6}$ layers [Fig. 3(d)]. This kind of layer-structured magnetic electride is also found in $\mathrm{Y}_{2} \mathrm{C}$ [45]. At the transformation to the $P 4 / \mathrm{mbm}$ structure, the ferromagnetism of $\mathrm{Y}_{3} \mathrm{~S}_{2}$ disappears deduced from the same profiles of spin-up and spin-down DOSs [Fig. 3(b)]. Simultaneously, no electron localizations are found in the interstices [Fig. 3(e)]. Different from that in $\mathrm{Y}_{3} \mathrm{~S}_{2}$, the contribution to the Fermi level in $\mathrm{YS}_{3}$ is mainly from $\mathrm{S}$ atoms, which is double that of $\mathrm{Y}$ atoms [Fig. 3(c)].
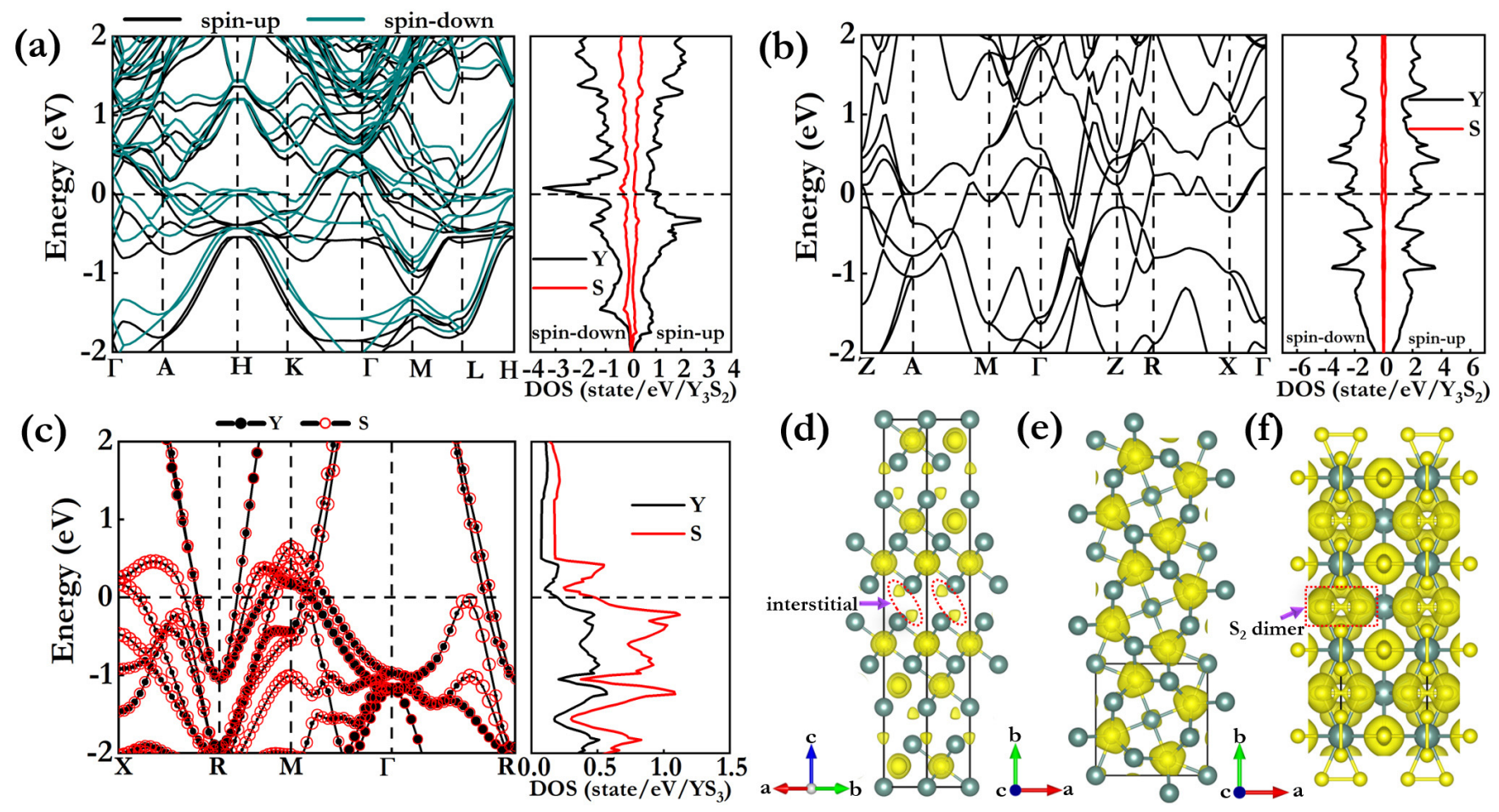

FIG. 3. The band structures and projected density of states (DOS) of the $R \overline{3} m$ structure of $\mathrm{Y}_{3} \mathrm{~S}_{2}$ at $6 \mathrm{GPa}$ (a), the $P 4 / m b m$ structure of $\mathrm{Y}_{3} \mathrm{~S}_{2}$ at $50 \mathrm{GPa}(\mathrm{b})$, and the $P m \overline{3}$ structure of $\mathrm{YS}_{3}$ at $50 \mathrm{GPa}$ (c). Bands in panel (a) are divided into spin up and spin down, and the bands in panel (c) are projected onto $\mathrm{Y}$ and $\mathrm{S}$ atoms with circle radii proportional to the weights of the corresponding atoms. [(d)-(f)]Three-dimensional ELF of the three structures with isosurface value of 0.7 . 


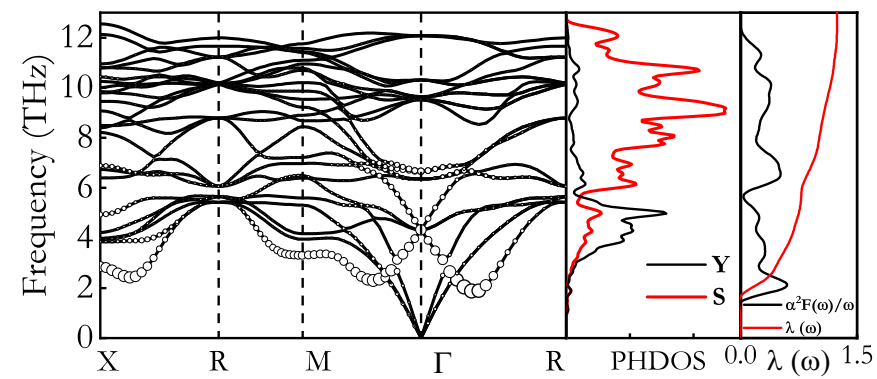

FIG. 4. The calculated phonon dispersion, projected phonon densities of states (PHDOS), Eliashberg spectral function $\left[\alpha^{2} F(\omega) /(\omega)\right]$, and EPC integration of $\lambda(\omega)$ for the $P m \overline{3}$ structure of $\mathrm{YS}_{3}$ at $50 \mathrm{GPa}$. Circles in the left panel indicate the phonon line width with radii proportional to the strength.

Bader analysis shows that each $\mathrm{Y}$ atom donates averagely 1.74 electrons to $\mathrm{S}$ atoms in $\mathrm{YS}_{3}$, much more than that (1.17) in $\mathrm{Y}_{3} \mathrm{~S}_{2}$ (Table S1). The large charge transfer from $\mathrm{Y}$ atoms to $\mathrm{S}$ atoms explains the primary contribution of $\mathrm{S}$ atom to the Fermi level in $\mathrm{YS}_{3}$.

The electron-phonon couplings (EPC) of $P 4 / m b m-Y_{3} \mathrm{~S}_{2}$ and $P m \overline{3}-Y_{3}$ were calculated to examine their potential superconductivity, while that of the $R \overline{3} m-\mathrm{Y}_{3} \mathrm{~S}_{2}$ was eliminated because of its magnetism. The electron-phonon coupling parameters $\lambda$ of $P 4 / \mathrm{mbm}-\mathrm{Y}_{3} \mathrm{~S}_{2}$ is calculated to be 0.48 at $50 \mathrm{GPa}$, the same as that of the known superconductor $\mathrm{Y}_{3} \mathrm{~S}_{4}$ (Table $\mathrm{S} 2$ ). The $T_{c}$ value was then estimated using the Allen-Dynes modified McMillan equation [40] using a typical Coulomb pseudopotential of $\mu^{*}=0.1$. Accordingly, the estimated $T_{c}$ of $P 4 / \mathrm{mbm}-\mathrm{Y}_{3} \mathrm{~S}_{2}$ is $\approx 2.9 \mathrm{~K}$, close to that (3.6 K [21]) of $\mathrm{Y}_{3} \mathrm{~S}_{4}$.

Strikingly, we find that $P m \overline{3}-\mathrm{YS}_{3}$ has a relatively larger $\lambda$ of 1.24 at $50 \mathrm{GPa}$, which leads to much higher $T_{c}$ of $18.5 \mathrm{~K}$ as compared to $\mathrm{Y}_{3} \mathrm{~S}_{2}$. Phonon dispersions and PHDOS show that the frequencies of $P m \overline{3}-Y_{3}$ can be divided into two parts, with the low frequencies $(<6 \mathrm{THz})$ dominated by vibrations of $\mathrm{Y}$ atoms, while the high end by vibrations of $\mathrm{S}$ atoms (Fig. 4). It is noteworthy that the low-frequency part contributes $64 \%$ to the total $\lambda$ and mainly originates from the contribution of three Kohn anomalies that locate at X-R, M- $\Gamma$, and $\Gamma$-R high-symmetry directions. Considering the dominant DOS of $\mathrm{S}$ atoms at the Fermi level, we therefore conclude that the high superconductivity of $\mathrm{YS}_{3}$ mainly originates from the coupling between the electrons of $\mathrm{S}$ atoms and phonon vibrations of $\mathrm{Y}$ atoms. The $T_{c}$ estimated for $\mathrm{YS}_{3}$ is comparable to the maximum $17 \mathrm{~K}$ for element $\mathrm{Y}$ (at $89.3 \mathrm{GPa}$ ) and $\mathrm{S}$ (at $160 \mathrm{GPa}$ ) but could be achieved at much lower pressure, indicating that alloying with other elements has the potential of decreasing the superconducting pressure. We have compared the volumes of $\mathrm{YS}_{3}$ with those of elemental Y plus $\mathrm{S}$ at different pressures and found the incorporation of $\mathrm{Y}$ atoms has precompression effects on the $\mathrm{S}$ sublattice. For example, the volume of $\mathrm{YS}_{3}$ at $50 \mathrm{GPa}$ is calculated to be $53.80 \AA^{3}$, which is much smaller than that $\left(61.74 \AA^{3}\right)$ of Y plus $S$ at the same pressure but is comparable to that $\left(53.92 \AA^{3}\right)$ at $160 \mathrm{GPa}$. In fact, this phenomenon is common in hydride superconductors, where the metallization can be achieved at lower pressure than with pure hydrogen, since the hydrogen

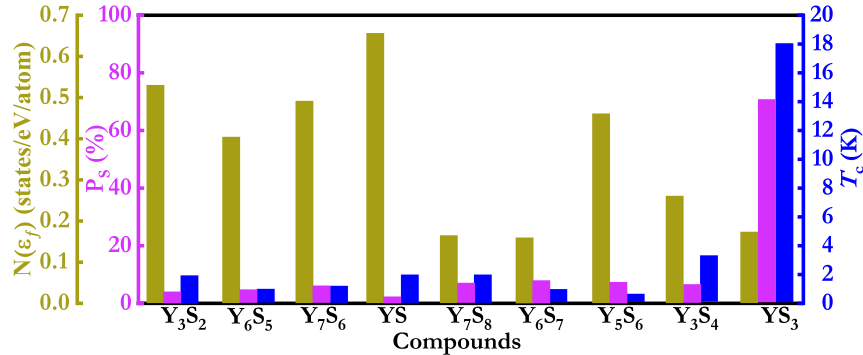

FIG. 5. The calculated $T_{c}$, the total DOS N $\left(\varepsilon_{f}\right)$ and the contribution of $\mathrm{S}$ atoms to the total DOS $\left(P_{S}\right)$ at the Fermi level for the stable Y-S compounds. $P_{S}$ is defined as the partial DOS of $\mathrm{S}$ atoms divided by the total DOS at the Fermi level.

atoms have already undergone chemical precompression by the heavy atoms $[25,34,48]$. Moreover, it is not unreasonable to deduce that hydrides of Y-S compounds have the potential to show high- $T_{c}$ superconductivity at lower pressures.

The superconductivities of other stable Y-S compounds were also examined and the estimated $T_{c}$ are summarized in Fig. 5. Unfortunately, all of them possess much lower superconductivities with $T_{c}$ less than $4 \mathrm{~K}$. Figure 5 also presents the total DOS as well as the contribution of $\mathrm{S}$ atoms to the total DOS at the Fermi level for all considered Y-S compounds. As compared to $\mathrm{YS}_{3}$, all other $\mathrm{Y}-\mathrm{S}$ compounds have comparable or even larger total DOS at the Fermi level. However, the contributions of S atoms to the total DOS in these compounds are negligible, completely distinct from the $75 \%$ contribution of $\mathrm{S}$ atoms in $\mathrm{YS}_{3}$. The antipodal contribution of $\mathrm{S}$ atoms to the total DOS may explain the relatively high superconductivity in $\mathrm{YS}_{3}$. In fact, such behaviors have also been observed in the case of superconducting metal hydrides, where a key characteristic is that a high $T_{c}$ is always accompanied by high contribution of hydrogen to the total DOS, such as in $\mathrm{LaH}_{10}$, $\mathrm{YH}_{10}[4-7,49-53]$.

\section{CONCLUSIONS}

In summary, the phase diagram of Y-S systems from atmospheric pressure to $50 \mathrm{GPa}$ is extensively explored by using a combination study of crystal structure predictions and first-principle calculations. The structure searches at ambient pressure have predicted five new stable compounds with stoichiometries $\mathrm{Y}_{7} \mathrm{~S}_{6}, \mathrm{Y}_{6} \mathrm{~S}_{5}, \mathrm{Y}_{7} \mathrm{~S}_{8}, \mathrm{Y}_{6} \mathrm{~S}_{7}$, and $\mathrm{Y}_{5} \mathrm{~S}_{6}$. Additionally, two pressure-stabilized compounds, $\mathrm{Y}_{3} \mathrm{~S}_{2}$ and $\mathrm{YS}_{3}$, are predicted to possess exotic properties. $\mathrm{Y}_{3} \mathrm{~S}_{2}$ becomes energetically stable at $6 \mathrm{GPa}$, which is found to be a layerstructured magnetic electride. The layered $\mathrm{Y}_{3} \mathrm{~S}_{2}$ transforms into a three-dimensional structure at $17 \mathrm{GPa}$ accompanied by the disappearance of magnetism and localized interstitial electrons. Interestingly, $\mathrm{YS}_{3}$ is predicted to be a potential superconductor with $T_{c}$ of $18.5 \mathrm{~K}$ at $50 \mathrm{GPa}$, close to the maximum value $(17 \mathrm{~K}$ ) observed in elemental $\mathrm{Y}$ at $89.3 \mathrm{GPa}$ and $\mathrm{S}$ at $160 \mathrm{GPa}$. It is notable that the pressure needed to realize the superconductivity in $\mathrm{YS}_{3}$ is much lower than those in corresponding elements, which means that it is much easier to achieve in experiments. Analysis shows that the coupling of electrons of $\mathrm{S}$ atoms and the vibrations of $\mathrm{Y}$ atoms mainly contribute to the superconductivity of $\mathrm{YS}_{3}$. The current results 
are expected to guide the future experimental study on Y-S compounds under pressure.

\section{ACKNOWLEDGMENTS}

The authors acknowledge funding from the National Natural Science Foundation of China under Grants No. 12074154 ,
No. 11722433, No. 11804128, and No. 11804129; the Six Talent Peaks Project and 333 High-Level Talents Project of Jiangsu Province; and the Science and Technology Project of Xuzhou under Grant No. KC19010. All the calculations were performed at the High Performance Computing Center of the School of Physics and Electronic Engineering of Jiangsu Normal University.
[1] A. Drozdov, M. Eremets, I. Troyan, V. Ksenofontov, and S. I. Shylin, Nature (London) 525, 73 (2015).

[2] I. Errea, M. Calandra, C. J. Pickard, J. Nelson, R. J. Needs, Y. Li, H. Liu, Y. Zhang, Y. Ma, and F. Mauri, Phys. Rev. Lett. 114, 157004 (2015).

[3] T. Jarlborg and A. Bianconi, Sci. Rep. 6, 24816 (2016).

[4] H. Liu, I. I. Naumov, R. Hoffmann, N. Ashcroft, and R. J. Hemley, Proc. Natl. Acad. Sci. USA 114, 6990 (2017).

[5] Z. M. Geballe, H. Liu, A. K. Mishra, M. Ahart, M. Somayazulu, Y. Meng, M. Baldini, and R. J. Hemley, Angew. Chem. 130, 696 (2018).

[6] H. Liu, I. I. Naumov, Z. M. Geballe, M. Somayazulu, J. S. Tse, and R. J. Hemley, Phys. Rev. B 98, 100102(R) (2018).

[7] I. Errea, F. Belli, L. Monacelli, A. Sanna, T. Koretsune, T. Tadano, R. Bianco, M. Calandra, R. Arita, F. Mauri et al., Nature (London) 578, 66 (2020).

[8] H. Wang, S. T. John, K. Tanaka, T. Iitaka, and Y. Ma, Proc. Natl. Acad. Sci. USA 109, 6463 (2012).

[9] Y. Li, J. Hao, H. Liu, S. T. John, Y. Wang, and Y. Ma, Sci. Rep. 5, 9948 (2015).

[10] D. Y. Kim, R. H. Scheicher, and R. Ahuja, Phys. Rev. Lett. 103, 077002 (2009).

[11] C. Heil, S. di Cataldo, G. B. Bachelet, and L. Boeri, Phys. Rev. B 99, 220502(R) (2019).

[12] H. Fujihisa, H. Yamawaki, M. Sakashita, A. Nakayama, T. Yamada, and K. Aoki, Phys. Rev. B 69, 214102 (2004).

[13] O. Zakharov and M. L. Cohen, Phys. Rev. B 52, 12572 (1995).

[14] V. V. Struzhkin, R. J. Hemley, H.-k. Mao, and Y. A. Timofeev, Nature (London) 390, 382 (1997).

[15] J. Michiels and K. Gschneidner, Jr., J. Alloys Compd. 247, 9 (1997).

[16] N. L. Eatough, A. W. Webb, and H. T. Hall, Inorg. Chem. 8, 2069 (1969).

[17] K. Range and R. Leeb, Z. Naturforsch., B 30, 889 (1975).

[18] B. Sahoo, K. Joshi, and S. C. Gupta, J. Appl. Phys. 115, 123502 (2014).

[19] P. Roedhammer, W. Reichardt, and F. Holtzberg, Phys. Rev. Lett. 40, 465 (1978).

[20] F. Hulliger and G. Hull Jr., Solid State Commun. 8, 1379 (1970).

[21] Y. Qi, Z. Xiao, J. Guo, H. Lei, T. Kamiya, and H. Hosono, EPL 121, 57001 (2018).

[22] W. Zhang, A. R. Oganov, A. F. Goncharov, Q. Zhu, S. E. Boulfelfel, A. O. Lyakhov, E. Stavrou, M. Somayazulu, V. B. Prakapenka, and Z. Konôpková, Science 342, 1502 (2013).

[23] J. Lin, Z. Zhao, C. Liu, J. Zhang, X. Du, G. Yang, and Y. Ma, J. Am. Chem. Soc. 141, 5409 (2019).

[24] M.-S. Miao, Nat. Chem. 5, 846 (2013).
[25] L. Zhang, Y. Wang, J. Lv, and Y. Ma, Nat. Rev. Mater. 2, 17005 (2017).

[26] C. Liu, X. Song, Q. Li, Y. Ma, and C. Chen, Phys. Rev. Lett. 124, 147001 (2020).

[27] Y. Wang, J. Lv, L. Zhu, and Y. Ma, Phys. Rev. B 82, 094116 (2010).

[28] Y. Wang, J. Lv, L. Zhu, and Y. Ma, Comput. Phys. Commun. 183, 2063 (2012).

[29] B. Gao, P. Gao, S. Lu, J. Lv, Y. Wang, and Y. Ma, Sci. Bull. 64, 301 (2019).

[30] J. Shi, W. Cui, S. Botti, and M. A. L. Marques, Phys. Rev. Mater. 2, 023604 (2018).

[31] J. Shi, W. Cui, J. Hao, M. Xu, X. Wang, and Y. Li, Nat. Commun. 11, 3164 (2020).

[32] W. Cui, T. Bi, J. Shi, Y. Li, H. Liu, E. Zurek, and R. J. Hemley, Phys. Rev. B 101, 134504 (2020).

[33] B. Liu, W. Cui, J. Shi, L. Zhu, J. Chen, S. Lin, R. Su, J. Ma, K. Yang, M. Xu, J. Hao, A. P. Durajski, J. Qi, Y. Li, and Y. Li, Phys. Rev. B 98, 174101 (2018).

[34] W. Cui and Y. Li, Chin. Phys. B 28, 107104 (2019).

[35] J. P. Perdew, K. Burke, and M. Ernzerhof, Phys. Rev. Lett. 80, 891 (1998).

[36] P. E. Blöchl, Phys. Rev. B 50, 17953 (1994).

[37] G. Kresse and J. Furthmüller, Comput. Mater. Sci. 6, 15 (1996).

[38] H. J. Monkhorst and J. D. Pack, Phys. Rev. B 13, 5188 (1976).

[39] P. Giannozzi, S. Baroni, N. Bonini, M. Calandra, R. Car, C. Cavazzoni, D. Ceresoli, G. L. Chiarotti, M. Cococcioni, I. Dabo et al., J. Phys.: Condens. Matter 21, 395502 (2009).

[40] R. Dynes, Solid State Commun. 10, 615 (1972).

[41] K. Momma and F. Izumi, J. Appl. Crystallogr. 44, 1272 (2011).

[42] Y. Chen, Q.-M. Hu, and R. Yang, Phys. Rev. Lett. 109, 157004 (2012).

[43] A. Jayaraman and R. Sherwood, Phys. Rev. 134, A691 (1964).

[44] B. Johansson and A. Rosengren, Phys. Rev. B 11, 2836 (1975).

[45] Y. Ge, S. Guan, and Y. Liu, New J. Phys. 19, 123020 (2017).

[46] See Supplemental Material at http://link.aps.org/supplemental/ 10.1103/PhysRevResearch.2.043435 for the structures, electronic properties, and phonon dispersions of five stable compounds at ambient pressure; the charge transfers and the structure information of seven stable compounds; and other details.

[47] S. Rettig and J. Trotter, Acta Crystallogr., Sec. C 43, 2260 (1987).

[48] N. W. Ashcroft, Phys. Rev. Lett. 92, 187002 (2004).

[49] A. Drozdov, P. Kong, V. Minkov, S. Besedin, M. Kuzovnikov, S. Mozaffari, L. Balicas, F. Balakirev, D. Graf, V. Prakapenka et al., Nature (London) 569, 528 (2019). 
[50] M. Somayazulu, M. Ahart, A. K. Mishra, Z. M. Geballe, M. Baldini, Y. Meng, V. V. Struzhkin, and R. J. Hemley, Phys. Rev. Lett. 122, 027001 (2019).

[51] F. Peng, Y. Sun, C. J. Pickard, R. J. Needs, Q. Wu, and Y. Ma, Phys. Rev. Lett. 119, 107001 (2017).
[52] H. Song, D. Duan, T. Cui, and V. Z. Kresin, Phys. Rev. B 102, 014510 (2020).

[53] A. M. Shipley, M. J. Hutcheon, M. S. Johnson, R. J. Needs, and C. J. Pickard, Phys. Rev. B 101, 224511 (2020). 